\title{
Effects of Magnetic Field and Nonlinear Temperature Profile on Marangoni Convection in Micropolar Fluid
}

\author{
M. N. Mahmud, ${ }^{1}$ R. Idris, ${ }^{2}$ and I. Hashim ${ }^{3}$ \\ ${ }^{1}$ Malaysian Institute of Chemical \& Bioengineering Technology, Universiti Kuala Lumpur, \\ 78000 Alor Gajah Melaka, Malaysia \\ ${ }^{2}$ Department of Mathematics, Faculty of Science $\mathcal{E}$ Technology, Universiti Malaysia Terengganu, \\ 21030 Kuala Terengganu, Malaysia \\ ${ }^{3}$ Centre for Modelling \& Data Analysis, School of Mathematical Sciences, Universiti Kebangsaan Malaysia, \\ 43600 UKM Bangi Selangor, Malaysia
}

Correspondence should be addressed to I. Hashim, ishak_h@ukm.my

Received 20 May 2009; Accepted 8 December 2009

Recommended by Tasawar K. Hayat

The combined effects of a uniform vertical magnetic field and a nonuniform basic temperature profile on the onset of steady Marangoni convection in a horizontal layer of micropolar fluid are studied. The closed-form expression for the Marangoni number $M$ for the onset of convection, valid for polynomial-type basic temperature profiles upto a third order, is obtained by the use of the single-term Galerkin technique. The critical conditions for the onset of convection have been presented graphically.

Copyright (C) 2009 M. N. Mahmud et al. This is an open access article distributed under the Creative Commons Attribution License, which permits unrestricted use, distribution, and reproduction in any medium, provided the original work is properly cited.

\section{Introduction}

Convective flow in a thin layer of fluid, free at the upper surface and heated from below, is of fundamental importance and a prototype to a more complex configuration in experiments and industrial processes. The convective flows in a liquid layer can be driven by buoyancy forces due to temperature gradients and/or thermocapillary (Marangoni) forces caused by surface tension gradients. Thermal convective problems have long been studied extensively since the pioneering experimental and theoretical works of Bénard [1], Rayleigh [2], and Pearson [3]. The instability problems have been studied in several other directions (cf. [418]).

Most of the previous studies were concerned with convection in Newtonian fluids. However, much less work has been done on convection in non-Newtonian fluids such 
as the micropolar fluids. The theory of micropolar fluids, as developed by Eringen [19], has been a field of sprightly research for the last few decades especially in many industrially important fluids like paints, polymeric suspensions, colloidal fluids, and also in physiological fluids such as normal human blood and synovial fluids. Rama Rao [20] studied the effect of a magnetic field on convection in a micropolar fluid. The onset of convection as overstable motions in a micropolar fluid was examined in [21]. Sharma and Gupta [22] studied convection in micropolar fluids in a porous medium. Ramdath [23] considered buoyancy-and thermocapillary-driven (Bénard-Marangoni) convection in a layer of micropolar fluid. The effect of throughflow on Marangoni convection in micropolar fluids was analyzed in [24]. Siddheshwar and Sri Krishna [25] presented both linear and nonlinear analyses of convection in a micropolar fluid occupying a porous medium. Sunil et al. [26] studied the effect of rotation on convection in a micropolar ferrofluid.

There has also been much less work focused on the effect of nonuniform temperature gradient on convection. Friedrich and Rudraiah [27] studied the combined effects of nonuniform temperature gradients and rotation on Marangoni convection. The combined effects of nonuniform temperature gradients and a magnetic field on Marangoni convection were investigated by Rudraiah et al. [28]. The work of Friedrich and Rudraiah [27] was further extended to include the effect of buoyancy by Rudraiah and Ramachandramurthy [29]. Dupont et al. [30] studied the effect of a cubic quasisteady temperature profile on Marangoni convection. The effects of nonuniform temperature gradients on the onset of oscillatory Marangoni and Bénard-Marangoni convection in a magnetic field were analyzed in [31, 32], respectively. Chiang [33] investigated the effect of Dupont et al. [30] temperature profile on the onset of stationary and oscillatory Bénard-Marangoni convection.

Thermal convection in micropolar fluids has also been studied. Rudraiah and Siddheshwar [34] analyzed the effects of nonuniform temperature gradients of parabolicand stepwise-types on the onset of Marangoni convection in a micropolar fluid. This study was later extended by Siddheshwar and Pranesh [35] to include the effect of a magnetic field and buoyancy forces. Very recently, Idris et al. [36] studied the effect of Dupont et al. [30] cubic temperature profile on the onset of Bénard-Marangoni convection in a micropolar fluid.

In this paper, we shall investigate the combined effects of Dupont et al. [30] cubic temperature profile and a magnetic field on the onset of Marangoni convection in a micropolar fluid. The single-term Galerkin technique [37] is employed to obtain a closedform expression of $M$ (Marangoni number) for the onset of convection. Comparisons with the other polynomial-type temperature profiles normally used by previous investigators shall be undertaken.

\section{Mathematical Formulation}

We wish to examine the stability of a horizontal layer of quiescent micropolar fluid of thickness $d$ in the presence of a magnetic field. We assume that the layer is bounded below by a rigid boundary, which is kept at a constant temperature, and above by a perfectly insulated, flat free surface. Moreover, the spin-vanishing boundary condition is assumed at the boundaries.

The governing equations for the problem are the continuity equation, conservation of momentum, conservation of angular momentum, conservation of energy, and magnetic 
induction, compare $[19,34,35]$ :

$$
\begin{gathered}
\nabla \cdot \vec{q}=0 \\
\rho_{0}\left[\frac{\partial \vec{q}}{\partial t}+(\vec{q} \cdot \nabla) \vec{q}\right]=-\nabla P+(2 \zeta+\eta) \nabla^{2} \vec{q}+\zeta \nabla \times \vec{w}+\mu_{m}(\vec{H} \cdot \nabla) \vec{H} \\
\rho_{0} I\left[\frac{\partial \vec{w}}{\partial t}+(\vec{q} \cdot \nabla) \vec{\omega}\right]=\left(\lambda^{\prime}+\eta^{\prime}\right) \nabla(\nabla \cdot \vec{w})+\eta^{\prime} \nabla^{2} \vec{w}+\zeta(\nabla \times \vec{q}-2 \vec{\omega}), \\
\frac{\partial T}{\partial t}+\left[\vec{q}-\frac{\beta}{\rho_{0} C_{v}} \nabla \times \vec{w}\right] \cdot \nabla T=x^{2} T \\
\frac{\partial \vec{H}}{\partial t}+(\vec{q} \cdot \nabla) \vec{H}=(\vec{H} \cdot \nabla) \vec{q}+\gamma_{m} \nabla^{2} \vec{H}
\end{gathered}
$$

where $\vec{q}$ is the velocity, $\vec{\omega}$ is the spin, $T$ is the temperature, $\vec{H}$ is the magnetic field, $P=$ $p+\mu_{m} H^{2} / 2$ is the hydromagnetic pressure, $\zeta$ is the coupling viscosity coefficient, $\eta$ is the shear kinematic viscosity coefficient, $I$ is the moment of inertia, $\lambda^{\prime}$ and $\eta^{\prime}$ are the bulk and shear spin viscosity coefficients, $\beta$ is the micropolar heat conduction coefficient, $C_{v}$ is the specific heat, $\chi$ is the thermal conductivity, and $\gamma_{m}=1 / \mu_{m} \sigma_{m}$ is the magnetic viscosity (where $\sigma_{m}$ electrical conductivity and $\mu_{m}$ magnetic permeability). All the viscosity coefficients, heat conduction coefficient and thermal conductivity are thermodynamically restricted on the assumption of Clausius-Duhem inequality (see Eringen [19]) and are all positive quantities.

The surface tension $\sigma$ at the free upper surface is

$$
\sigma=\sigma_{0}-\sigma_{1}\left(T-T_{0}\right)
$$

where $\sigma_{0}$ is the unperturbed value of $\sigma$ and $\sigma_{1}=-(\mathrm{d} \sigma / \mathrm{d} T)_{T_{0}}$. The perturbation (2.1) are nondimensionalised using the following definition:

$$
\begin{aligned}
& \left(x^{*}, y^{*}, z^{*}\right)=\frac{(x, y, z)}{d}, \quad \vec{q}^{*}=\frac{\vec{q}^{\prime}}{x / d^{\prime}} \\
& \vec{\omega}^{*}=\frac{\vec{\omega}^{\prime}}{x / d^{2}}, \quad T^{*}=\frac{T^{\prime}}{\Delta T}, \quad \vec{H}^{*}=\frac{\vec{H}^{\prime}}{H_{0}} .
\end{aligned}
$$


Following the classical lines of linear stability theory, the linearised and dimensionless governing equations are

$$
\begin{gathered}
\left(1+N_{1}\right) \nabla^{4} W+N_{1} \nabla^{2} \Omega_{z}+Q \frac{P r}{P m} \nabla^{2}\left(\frac{\partial H_{z}}{\partial z}\right)=0 \\
N_{3} \nabla^{2} \Omega_{z}-2 N_{1} \Omega_{z}-N_{1} \nabla^{2} W=0 \\
\nabla^{2} \Theta+f(z)\left(W-N_{5} \Omega_{z}\right)=0 \\
\nabla^{2} H_{z}+\frac{P m}{P r} \frac{\partial W}{\partial z}=0
\end{gathered}
$$

where $W, \Omega_{z}, \Theta$, and $H_{z}$ are, respectively, the amplitudes of the infinitesimal perturbations of velocity, spin, temperature, and magnetic field, $N_{1}=\zeta /(\zeta+\eta)$ is the coupling parameter $\left(0 \leq N_{1} \leq 1\right), N_{3}=\eta^{\prime} /(\zeta+\eta)$ is the couple stress parameter $\left(0 \leq N_{3} \leq m\right.$, $m$ : finite, real), $N_{5}=\beta /\left(\rho_{0} C_{v} d^{2}\right)$ is the micropolar heat conduction parameter $\left(0 \leq N_{5} \leq n, n\right.$ : finite, real), $Q=\mu_{m} H_{0}^{2} d^{2} /\left[(\zeta+\eta) \gamma_{m}\right]$ is the Chandrasekhar number, $\operatorname{Pr}=(\zeta+\eta) / X$ is the Prandtl number, $P m=(\zeta+\eta) / \gamma_{m}$ is the magnetic Prandtl number, and $f(z)$ is a nondimensional basic temperature gradient satisfying the condition $\int_{0}^{1} f(z) \mathrm{d} z=1$.

The infinitesimal perturbations $W, \Omega_{z}, \Theta$, and $H_{z}$ are assumed to be periodic waves and hence these permit a normal mode solution in the following form:

$$
\left[W, \Omega_{z}, \Theta, H_{z}\right]=\left[W(z), \Omega_{z}(z), \Theta(z), H_{z}(z)\right] \exp [i(l x+m y)],
$$

where $l$ and $m$ are horizontal components of the wave number $\vec{a}$.

Substituting (2.5) into (2.4), we get

$$
\begin{gathered}
\left(1+N_{1}\right)\left(D^{2}-a^{2}\right)^{2} W+N_{1}\left(D^{2}-a^{2}\right) \Omega+Q \frac{P r}{P m}\left(D^{2}-a^{2}\right) D H_{z}=0, \\
N_{1}\left(D^{2}-a^{2}\right) W-N_{3}\left(D^{2}-a^{2}\right) \Omega+2 N_{1} \Omega=0, \\
\left(D^{2}-a^{2}\right) \Theta+f(z)\left(W-N_{5} \Omega\right)=0, \\
\left(D^{2}-a^{2}\right) H_{z}+\frac{P m}{P r} D W=0,
\end{gathered}
$$

where $D \equiv \mathrm{d} / \mathrm{d} z$.

Eliminating $H_{z}$ between (2.6) and (2.9), we obtain

$$
\left(1+N_{1}\right)\left(D^{2}-a^{2}\right)^{2} W+N_{1}\left(D^{2}-a^{2}\right) \Omega-Q D^{2} W=0 .
$$

Equations (2.7), (2.8), and (2.10) are solved subject to the linearized and dimensionless boundary conditions:

$$
\begin{gathered}
W=D^{2} W+a^{2} M \Theta=D \Theta=\Omega=0 a t z=1, \\
W=D W=\Theta=\Omega=0 a t z=0,
\end{gathered}
$$


Table 1: Reference steady-state temperature gradients.

\begin{tabular}{clcccc}
\hline Model & $\begin{array}{l}\text { Reference steady-state } \\
\text { temperature gradient }\end{array}$ & $f(z)$ & $a_{1}^{*}$ & $a_{2}^{*}$ & $a_{3}^{*}$ \\
\hline 1 & Linear & 1 & 1 & 0 & 0 \\
2 & Inverted parabolic & $2(1-z)$ & 0 & -1 & 0 \\
3 & Cubic 1 & $3(z-1)^{2}$ & 0 & 0 & 1 \\
4 & Cubic 2 & $0.6+1.02(z-1)^{2}$ & 0.6 & 0 & 0.34 \\
\hline
\end{tabular}

where $M=\sigma_{1} \Delta T d / \mu \chi$ is the Marangoni number (where $\Delta T$ is the temperature difference between the two boundaries).

Following [30], we consider the steady state temperature profile given by

$$
\bar{T}_{b}=\bar{T}_{O S}-a_{1}(\bar{z}-d)-a_{2}(\bar{z}-d)^{2}-a_{3}(\bar{z}-d)^{3},
$$

which precisely represents an experimental data, where $\left(^{-}\right)$denotes dimensional quantities, $\bar{T}_{O S}$ is the temperature at the upper free surface, and $a_{i}, i=1,2,3$ are constants. In nondimensional form, the $f(z)$ in this case is given by

$$
f(z)=a_{1}^{*}+2 a_{2}^{*}(z-1)+3 a_{3}^{*}(z-1)^{2} .
$$

The case $a_{1}^{*}=1, a_{2}^{*}=0$, and $a_{3}^{*}=0$ recovers the classical linear basic state temperature distribution. The different temperature gradients studied in this paper are listed in Table 1.

\section{Solution of the Linearized Problem}

Equations (2.7), (2.8), and (2.10) subject to the boundary conditions (2.11) constitute an eigenvalue problem. To solve the resulting eigenvalue problem, a single-term Galerkin expansion technique [37] is used to encompass a vast parameter space. Also, the technique employed yields sufficiently accurate and useful results for the purpose in hand with minimum of mathematics [37].

First we multiply (2.7), (2.8) and (2.10) by $\Omega, \Theta$, and $W$, respectively. Then we integrate the resulting equations by parts with respect to $z$ from 0 to 1 . By using the boundary conditions (2.11) and taking $\Omega=A \Omega_{1}(z), \Theta=B \Theta_{1}(z)$, and $W=C W_{1}(z)$, and in which $A, B$, and $C$ are constants and $\Omega_{1}(z)=z(1-z), \Theta_{1}(z)=z(2-z)$, and $W_{1}(z)=z^{2}\left(1-z^{2}\right)$ are trial functions, yields the eigenvalue $M$ in the form

$$
M=\frac{\left[\left\langle\left(D \theta_{1}\right)^{2}\right\rangle+a^{2}\left\langle\theta_{1}^{2}\right\rangle\right]\left[C_{1}\left(C_{2}-Q\left\langle\left(D W_{1}\right)^{2}\right\rangle\right)+N_{1}^{2} C_{3}^{2}\right]}{\left(1+N_{1}\right) a^{2} \theta(1) D W(1) C_{4}}
$$


where

$$
\begin{gathered}
C_{1}=N_{3}\left\langle\left(D \Omega_{1}\right)^{2}\right\rangle+\left(N_{3} a^{2}+2 N_{1}\right)\left\langle\Omega_{1}^{2}\right\rangle, \\
C_{2}=-\left(1+N_{1}\right)\left[\left\langle\left(D^{2} W_{1}\right)^{2}\right\rangle+2 a^{2}\left\langle\left(D W_{1}\right)^{2}\right\rangle+a^{4}\left\langle W_{1}^{2}\right\rangle\right] \\
C_{3}=\left\langle\left(D \Omega_{1}\right)\left(D W_{1}\right)\right\rangle+a^{2}\left\langle W_{1} \Omega_{1}\right\rangle \\
C_{4}=\left\langle f(z) W_{1} \theta_{1}\right\rangle C_{1}-N_{1} N_{5}\left\langle f(z) \theta_{1} \Omega_{1}\right\rangle C_{3} .
\end{gathered}
$$

Now with $f(z)$ as given in (2.13), we rewrite the expression (3.1) in the closed-form expression for $M$ :

$$
M=\frac{f_{4}\left[f_{2}\left\{315\left(1+N_{1}\right) f_{3}+132 Q\right\}-315 f_{1}^{2}\right]}{630\left(1+N_{1}\right)\left[f_{2} f_{6}-N_{5} f_{1} f_{5}\right]}
$$

where

$$
\begin{gathered}
f_{1}=\frac{1}{15} N_{1}\left(4+\frac{11}{28} a^{2}\right), \quad f_{2}=\frac{1}{3}\left(N_{3}+\frac{1}{10} N_{3} a^{2}+\frac{1}{5} N_{1}\right), \\
f_{3}=\frac{4}{5}\left(21+\frac{22}{21} a^{2}+\frac{2}{63} a^{4}\right), \quad f_{4}=\frac{4}{3}\left(1+\frac{2}{5} a^{2}\right), \\
f_{5}=\frac{1}{10}\left(\frac{11}{14} a_{3}^{*}-a_{2}^{*}+\frac{7}{6} a_{1}^{*}\right) a^{2}, \quad f_{6}=\frac{1}{21}\left(a_{3}^{*}-\frac{31}{20} a_{2}^{*}+\frac{23}{10} a_{1}^{*}\right) a^{2} .
\end{gathered}
$$

We remark that (3.3) is valid for all polynomial-type basic temperature profiles up to a third order. The critical Marangoni number, $M_{c}$, for the onset of convection is the global minimum of $M$ over $a \geq 0$.

\section{Discussion}

The critical Marangoni number $M_{c}$ which attains its minimum at $a_{c}^{2}$ is computed from (3.3) for different volumes of $Q, N_{1}, N_{3}$, and $N_{5}$ and the results are depicted in Figures 1, 2, and 3 . We recover the results of Rudraiah and Siddheshwar [34] for the linear and inverted parabolic temperature gradients when $Q=0$. We observe that as $N_{1}$ or $N_{5}$ increases, $M_{c}$ also increases. Obviously, the onset of convection will be delayed by increasing the concentration of the microelements or heat induced into the fluid by the microelements. But, an increase in $N_{3}$ leads to a decrease in microrotation, and hence the system becomes more unstable. Also it is observed that Model 4 (Cubic 2), with $a_{1}^{*}=0.6, a_{2}^{*}=0, a_{3}^{*}=0.34$ as used by Dupont et al. [30], is less stabilizing than Model 2 (Inverted parabolic), that is, $M_{c 4}<M_{c 2}$. Based on our results, Model 3 (Cubic 1 ) with $a_{1}^{*}=0, a_{2}^{*}=0, a_{3}^{*}=1$ is shown to be the most stabilizing of all the considered types of temperature gradients, that is, $M_{c 1}<M_{c 4}<M_{c 2}<M_{c 3}$.

Figures 4-6 illustrate the variations of the critical Marangoni number $M_{c}$ with the Chandrasekhar number $Q$ for some assigned values of $N_{1}, N_{3}$, and $N_{5}$, respectively. The 


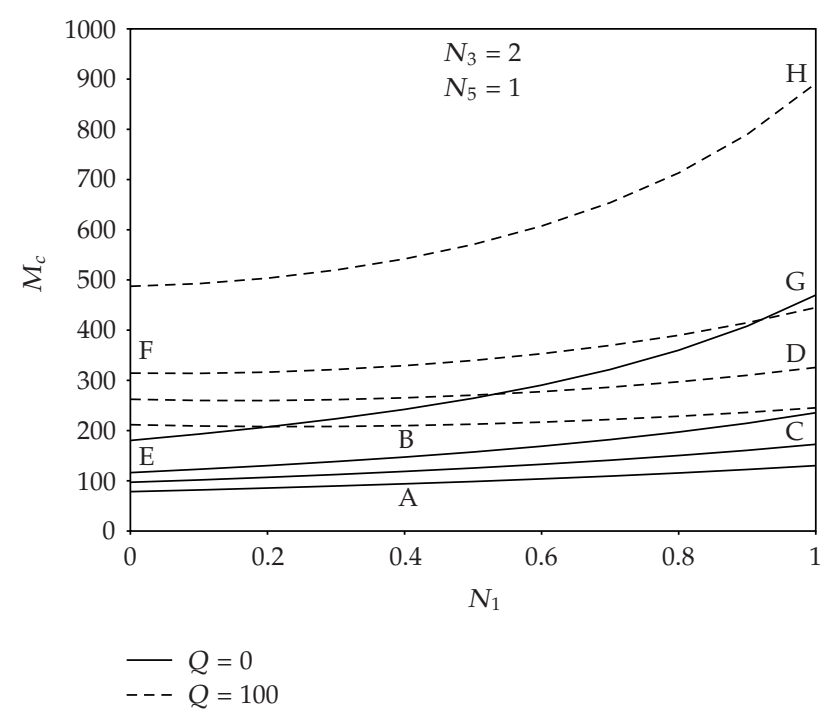

Figure 1: Plot of $M_{c}$ versus $N_{1}$ with $N_{3}=2$ and $N_{5}=1$, A: Linear. $Q=0$; B: Linear, $Q=100$; C: Cubic 2, $Q=0$; D: Cubic 2, $Q=100$; E: Inv. Parabolic, $Q=0$; F: Inv. Parabolic, $Q=100$; G: Cubic 1, $Q=0$; H: Cubic $1, Q=100$.

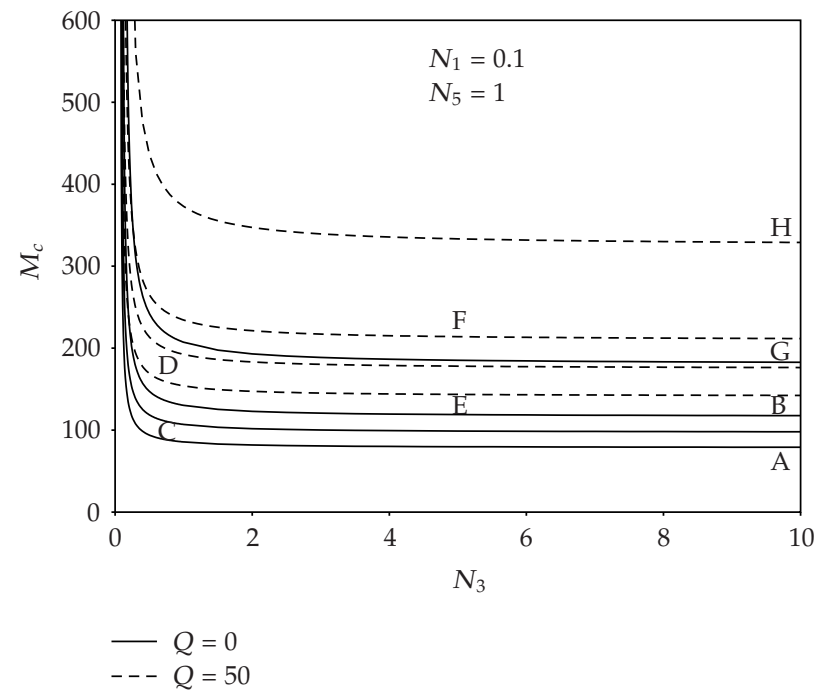

Figure 2: Plot of $M_{c}$ versus $N_{3}$ with $N_{1}=0.1$ and $N_{5}=1.0$, A: Linear, $Q=0$; B: Linear, $Q=50$; C: Cubic 2, $Q=0$; D: Cubic 2, $Q=50$; E: Inv. Parabolic, $Q=0$; F: Inv. Parabolic, $Q=50$, G: Cubic $1, Q=0$, H: Cubic 1 , $Q=50$.

results indicate that $M_{c}$ is generally an increasing function of $Q$. From Figure 4, we notice that the increase in the concentration of the microelements is to stabilize the system by superposing on the effect of the magnetic field. Figure 5 shows that the effect of $N_{3}$ on the system is very small compared to the effects of the other microelements. As before, Model 3 (Cubic 1) with $a_{1}^{*}=0, a_{2}^{*}=0, a_{3}^{*}=1$ is shown to be the most stabilizing of all the considered types of temperature gradients, that is, $M_{c 1}<M_{c 4}<M_{c 2}<M_{c 3}$. 


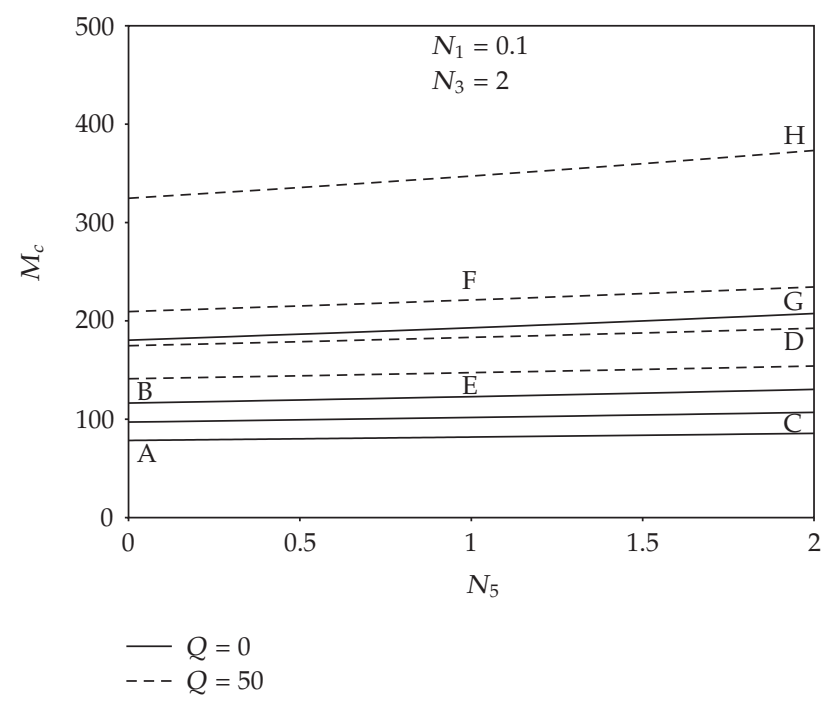

Figure 3: Plot of $M_{c}$ versus $N_{5}$ with $N_{1}=0.1$ and $N_{3}=2.0$, A: Linear, $Q=0$; B: Linear, $Q=50$; C: Cubic 2, $Q=0$; D: Cubic 2, $Q=50$; E: Inv. Parabolic, $Q=0$; F: Inv. Parabolic, $Q=50$; G: Cubic 1, $Q=0$; H: Cubic 1 , $Q=50$.

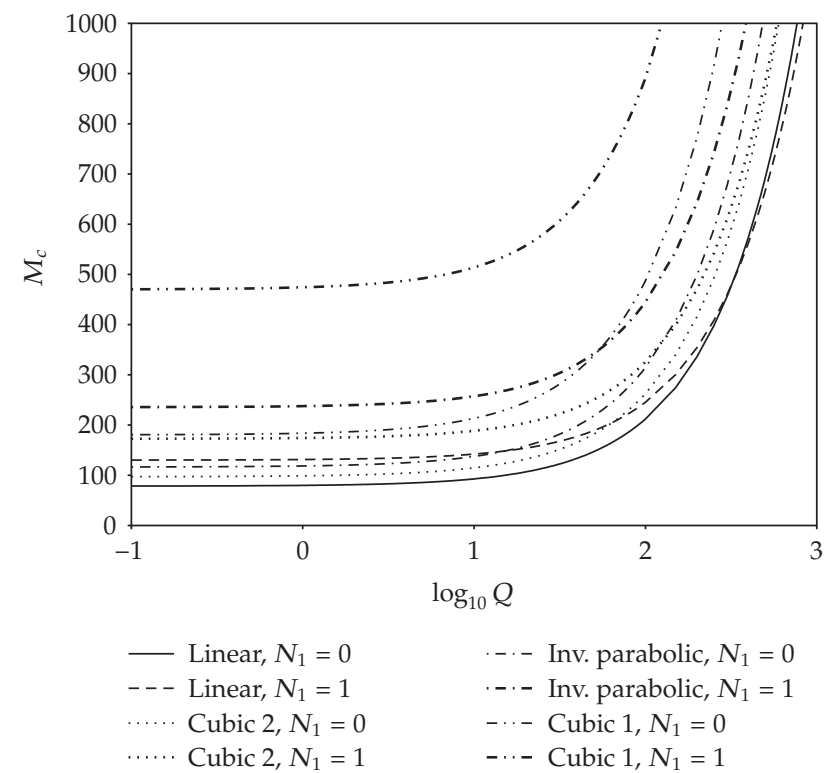

Figure 4: Plot of $M_{c}$ versus $Q$ for different temperature gradients with $N_{3}=2.0$ and $N_{5}=1.0$.

\section{Conclusion}

The problem of Marangoni convection in a micropolar fluid in the presence of a cubic basic state temperature profile and a vertical magnetic field has been studied theoretically. The results indicate that it is possible to delay the onset of convection by the application of a cubic 


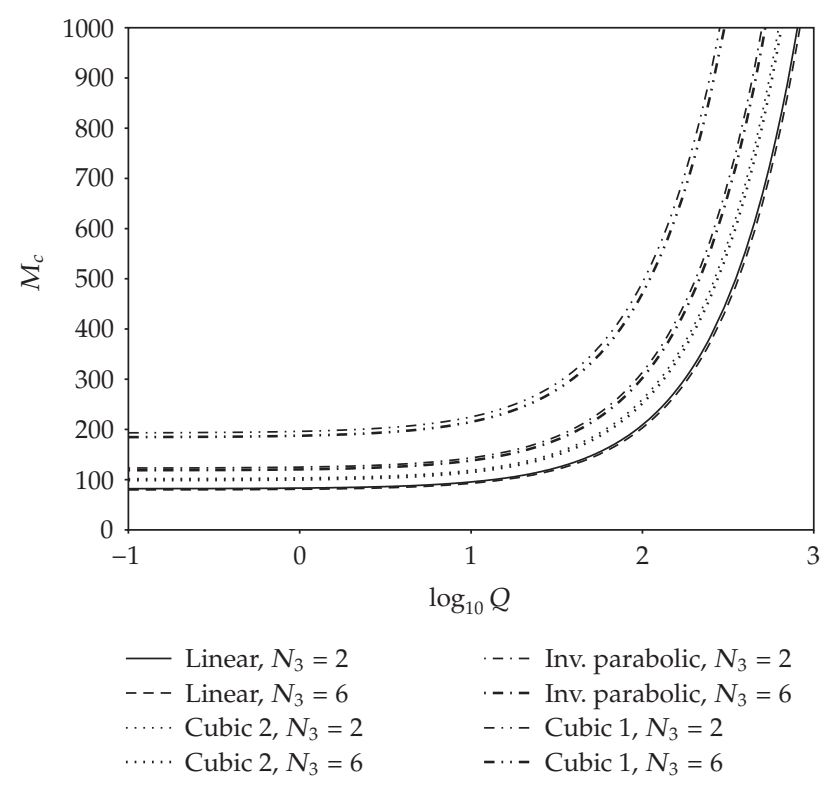

Figure 5: Plot of $M_{c}$ versus $Q$ for different temperature gradients with $N_{1}=0.1$ and $N_{5}=1.0$.

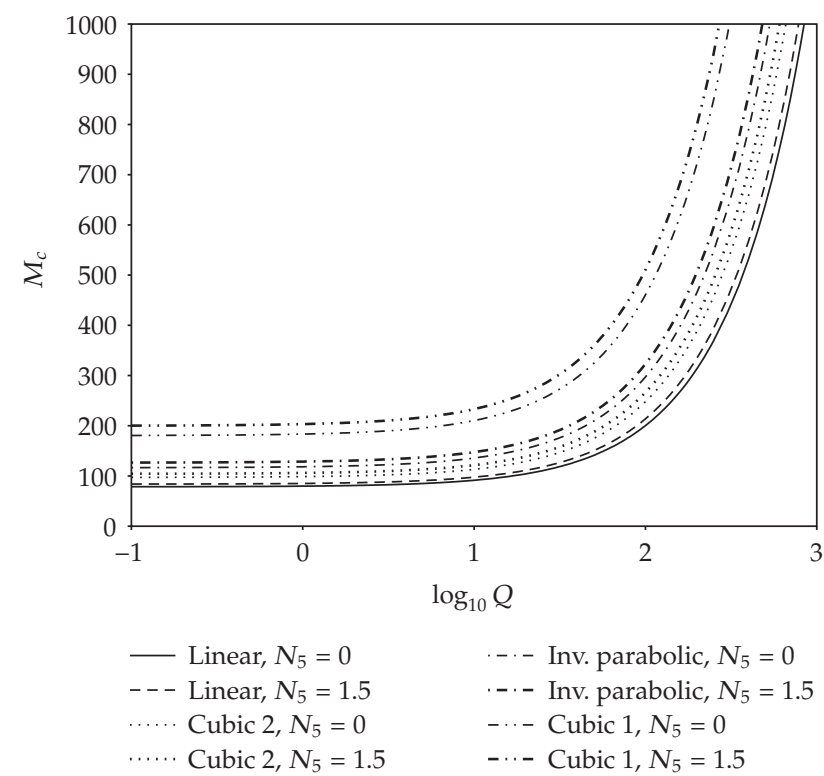

Figure 6: Plot of $M_{c}$ versus $Q$ for different temperature gradients with $N_{1}=0.1$ and $N_{3}=2.0$.

basic state temperature profile. In addition, the presence of a magnetic field is to suppress Magnetomarangoni convection and hence leads to a more stable system. As expected, the presence of the micron-sized suspended particles adds to the stabilizing effect of the magnetic field. 


\section{Acknowledgment}

The authors acknowledge the financial support received under the Grant UKM-GUP-BTT-0725-173 and from Universiti Kuala Lumpur (UniKL MICET).

\section{References}

[1] H. Bénard, "Les tourbillons cellulaires dans une nappe liquide," Revue Générale des Sciences Pures et Appliquées, vol. 11, pp. 1261-1271, 1900.

[2] L. Rayleigh, "On convection currents in a horizontal layer of fluid when the higher temperature is on the other side," Philosophical Magazine, vol. 32, pp. 529-546, 1916.

[3] J. R. A. Pearson, "On convection cells induced by surface tension," Journal of Fluid Mechanics, vol. 4, pp. 489-500, 1958.

[4] S. Chandrasekhar, Hydrodynamic and Hydromagnetic Stability, The International Series of Monographs on Physics, Clarendon Press, Oxford, UK, 1961.

[5] D. A. Nield, "Surface tension and buoyancy effects in the cellular convection of an electrically conducting liquid in a magnetic field," Zeitschrift für Angewandte Mathematik und Physik, vol. 17, no. 1, pp. 131-139, 1966.

[6] M. Takashima, "Nature of the neutral state in convective instability induced by surface tension and buoyancy," Journal of the Physical Society of Japan, vol. 28, p. 810, 1970.

[7] S. H. Davis and G. M. Homsy, "Energy stability theory for free-surface problems: buoyancythermocapillary layers," Journal of Fluid Mechanics, vol. 98, no. 3, pp. 527-553, 1980.

[8] M.-I. Char and K.-T. Chiang, "Boundary effects on the Bénard-Marangoni instability under an electric field," Applied Scientific Research, vol. 52, no. 4, pp. 331-354, 1994.

[9] I. Hashim and S. K. Wilson, "The effect of a uniform vertical magnetic field on the onset of oscillatory Marangoni convection in a horizontal layer of conducting fluid," Acta Mechanica, vol. 132, no. 1-4, pp. 129-146, 1999.

[10] I. Hashim, "On competition between modes at the onset of Bénard-Marangoni convection in a layer of fluid," The Australian \& New Zealand Industrial and Applied Mathematics Journal, vol. 43, no. 3, pp. 387-395, 2002.

[11] I. Hashim and N. Md Arifin, "The effect of a magnetic field on the linear growth rates of BénardMarangoni convection," Microgravity Science and Technology, vol. 17, no. 2, pp. 5-8, 2005.

[12] I. Hashim and Z. Siri, "Stabilization of steady and oscillatory marangoni instability in rotating fluid layer by feedback control strategy," Numerical Heat Transfer A, vol. 54, no. 6, pp. 647-663, 2008.

[13] S. Awang Kechil and I. Hashim, "Control of Marangoni instability in a layer of variable-viscosity fluid," International Communications in Heat and Mass Transfer, vol. 35, no. 10, pp. 1368-1374, 2008.

[14] Z. Siri and I. Hashim, "Control of oscillatory Marangoni convection in a rotating fluid layer," International Communications in Heat and Mass Transfer, vol. 35, no. 9, pp. 1130-1133, 2008.

[15] S. Awang Kechil and I. Hashim, "Oscillatory Marangoni convection in variable-viscosity fluid layer: the effect of thermal feedback control," International Journal of Thermal Sciences, vol. 48, no. 6, pp. 11021107, 2009.

[16] W.-M. Yang, "Thermal instability of a fluid layer induced by radiation," Numerical Heat Transfer A, vol. 17, no. 3, pp. 365-376, 1990.

[17] A. Y. Gelfgat and I. Tanasawa, "Numerical analysis of oscillatory instability of buoyancy convection with the Galerkin spectral method," Numerical Heat Transfer A, vol. 25, no. 6, pp. 627-648, 1994.

[18] E. Evren-Selamet, V. S. Arpaci, and A. T. Chai, “Thermocapillary-driven flow past the Marangoni instability," Numerical Heat Transfer A, vol. 26, no. 5, pp. 521-535, 1994.

[19] A. C. Eringen, "Micropolar theory of liquid crystals," in Liquid Crystals and Ordered Fluids, J. F. Johnson and R. S. Porter, Eds., vol. 3, Plenum, New York, NY, USA, 1978.

[20] K. V. Rama Rao, "Thermal instability in a micropolar fluid layer subject to a magnetic field," International Journal of Engineering Science, vol. 18, no. 5, pp. 741-750, 1980.

[21] C. Pérez-García and J. M. Rubí, “On the possibility of overstable motions of micropolar fluids heated from below," International Journal of Engineering Science, vol. 20, no. 7, pp. 873-878, 1982. 
[22] R. C. Sharma and U. Gupta, "Thermal convection in micropolar fluids in porous medium," International Journal of Engineering Science, vol. 33, no. 13, pp. 1887-1892, 1995.

[23] G. Ramdath, "Benard-Marangoni instability in a layer of micropolar fluid," Journal of Non-Equilibrium Thermodynamics, vol. 22, no. 4, pp. 299-310, 1997.

[24] Y. N. Murty and V. V. Ramana Rao, "Effect of throughflow on Marangoni convection in micropolar fluids," Acta Mechanica, vol. 138, no. 3, pp. 211-217, 1999.

[25] P. G. Siddheshwar and C. V. Sri Krishna, "Linear and non-linear analyses of convection in a micropolar fluid occupying a porous medium," International Journal of Non-Linear Mechanics, vol. 38, no. 10, pp. 1561-1579, 2003.

[26] Sunil, P. Chand, P. K. Bharti, and A. Mahajan, "Thermal convection in micropolar ferrofluid in the presence of rotation," Journal of Magnetism and Magnetic Materials, vol. 320, no. 3-4, pp. 316-324, 2008.

[27] R. Friedrich and N. Rudraiah, "Marangoni convection in a rotating fluid layer with non-uniform temperature gradient," International Journal of Heat and Mass Transfer, vol. 27, no. 3, pp. 443-449, 1984.

[28] N. Rudraiah, V. Ramachandramurthy, and O. P. Chandna, "Effects of magnetic field and non-uniform temperature gradient on Marangoni convection," International Journal of Heat and Mass Transfer, vol. 28 , no. 8, pp. 1621-1624, 1985.

[29] N. Rudraiah and V. Ramachandramurthy, "Effects of non-uniform temperature gradient and Coriolis force on Bénard-Marangoni's instability," Acta Mechanica, vol. 61, no. 1-4, pp. 37-50, 1986.

[30] O. Dupont, M. Hennenberg, and J. C. Legros, "Marangoni-Bénard instabilities under non-steady conditions. Experimental and theoretical results," International Journal of Heat and Mass Transfer, vol. 35, no. 12, pp. 3237-3244, 1992.

[31] M.-I. Char and C.-C. Chen, "Effects of nonuniform temperature gradients on the onset of oscillatory Marangoni convection in a magnetic field," Acta Mechanica, vol. 161, no. 1-2, pp. 17-30, 2003.

[32] M.-I. Char and C.-C. Chen, "Effect of a non-uniform temperature gradient on the onset of oscillatory Bénard-Marangoni convection of an electrically conducting liquid in a magnetic field," International Journal of Engineering Science, vol. 41, no. 15, pp. 1711-1727, 2003.

[33] K.-T. Chiang, "Effect of a non-uniform basic temperature gradient on the onset of Bénard-Marangoni convection: stationary and oscillatory analyses," International Communications in Heat and Mass Transfer, vol. 32, no. 1-2, pp. 192-203, 2005.

[34] N. Rudraiah and P. G. Siddheshwar, "Effect of non-uniform basic temperature gradient on the onset of Marangoni convection in a fluid with suspended particles," Aerospace Science and Technology, vol. 4, no. 8, pp. 517-523, 2000.

[35] P. G. Siddheshwar and S. Pranesh, "Magnetoconvection in fluids with suspended particles under $1 \mathrm{~g}$ and $\mu \mathrm{g}$," Aerospace Science and Technology, vol. 6, no. 2, pp. 105-114, 2002.

[36] R. Idris, H. Othman, and I. Hashim, "On effect of non-uniform basic temperature gradient on BénardMarangoni convection in micropolar fluid," International Communications in Heat and Mass Transfer, vol. 36, no. 3, pp. 255-258, 2009.

[37] B. A. Finlayson, The Method of Weighted Residuals and Variational Principles, Academic Press, New York, NY, USA, 1972 


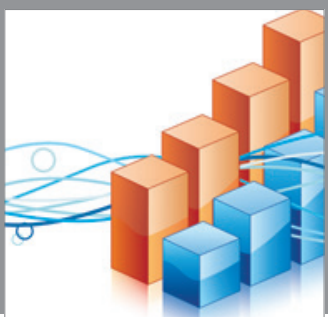

Advances in

Operations Research

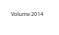

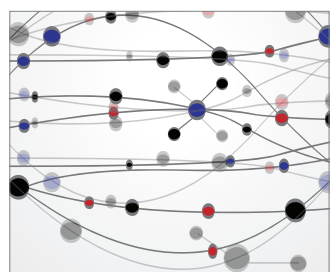

\section{The Scientific} World Journal
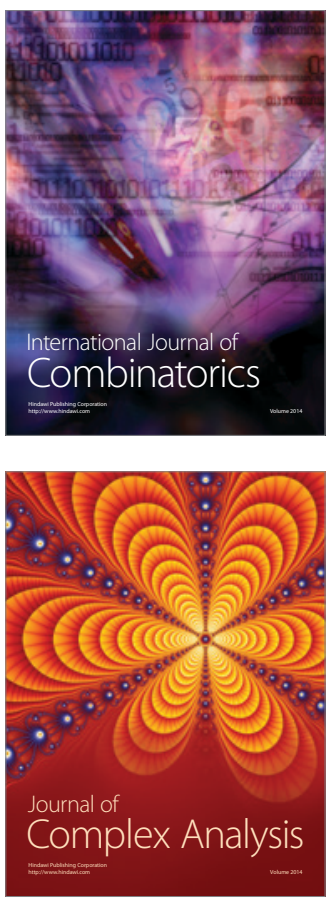

International Journal of

Mathematics and

Mathematical

Sciences
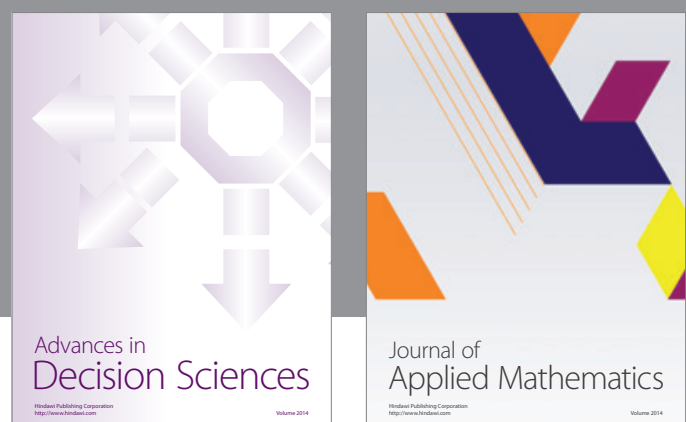

Journal of

Applied Mathematics
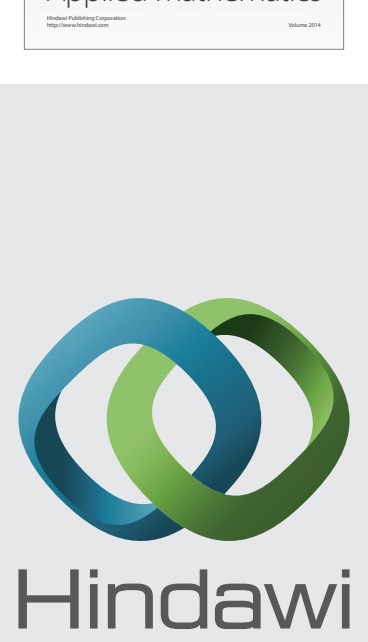

Submit your manuscripts at http://www.hindawi.com
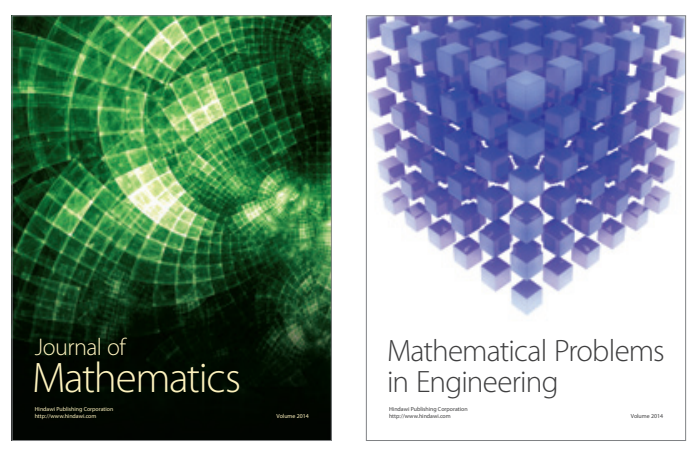

Mathematical Problems in Engineering
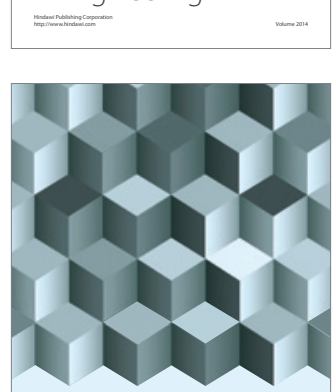

Journal of

Function Spaces
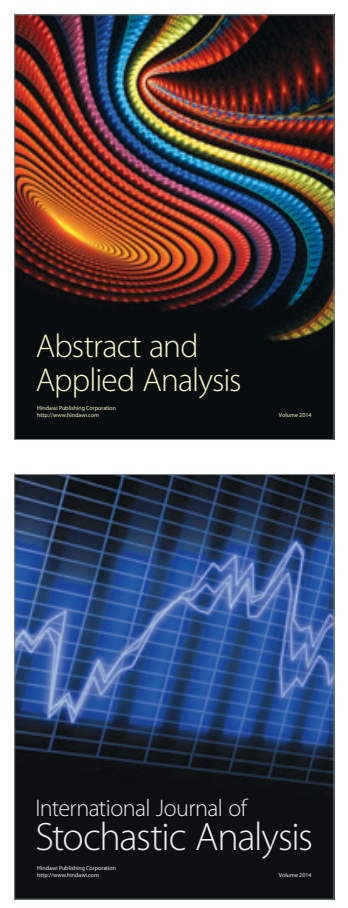

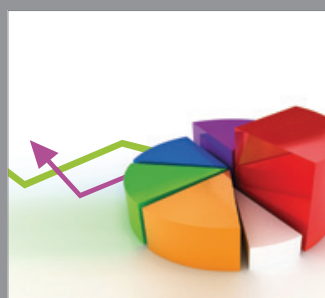

ournal of

Probability and Statistics

Promensencen
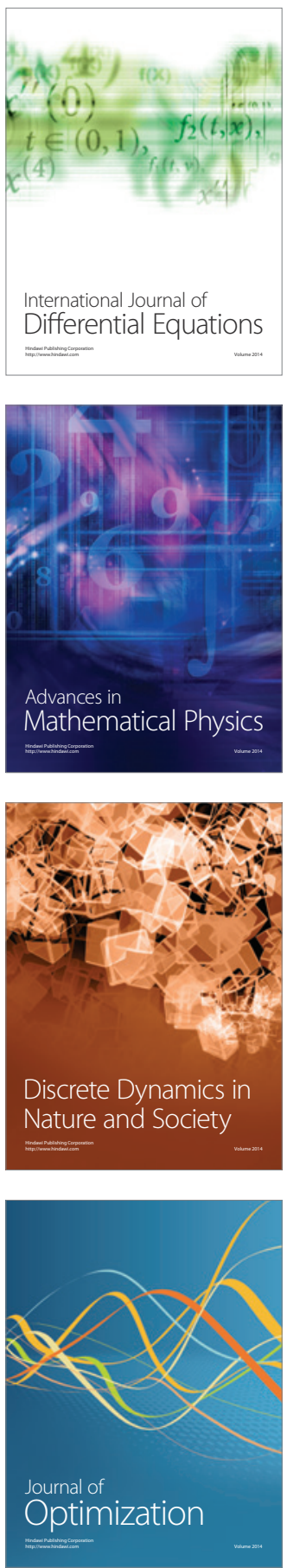